\title{
Semiclassical Husimi Function of Simple and Chaotic Systems
}

\author{
Adélcio C. Oliveira \\ Departamento de Física e Matemática, Universidade Federal de São João Del Rei, Ouro Branco, Brazil \\ Email: adelcio@ufsj.edu.br
}

Received April 10, 2012; revised May 5, 2012; accepted May 31, 2012

\begin{abstract}
We review the semiclassical method proposed in [1], a generalization of this method for n-dimensional system is presented. Using the cited method, we present an analytical method of obtain the semiclassical Husimi Function. The validity of the method is tested using Harmonic Oscillator, Morse Potential and Dikie's Model as example, we found a good accuracy in the classical limit.
\end{abstract}

Keywords: Classical Limit; Husimi Function; Quantum Chaos

\section{Introduction}

Since early times of quantum theory, some quantization difficulties of non integrable systems were pointed by Einstein [2,3]. Recently, due to the pioneer discoveries of classically chaotic systems, the subject has yielded many interesting and important results both from the point of view of numerical models and (not as many) analytical proofs [4-6]. Also in this direction, the phenomena of scar [7-11] drew much attention. They showed that the Hamiltonians eigenfunctions of chaotic systems exhibit "scars" around unstable periodic orbit. An question that appears from those analyses is related to chaotic manifestation of classical chaos over the eigenfunctions in terms of quantities that are base independent [12-15]. In opposition, it has been reported that scars can exist in regions where there are no periodic orbits [16]. The search for classical "imprints" than the celebrated phenomena of scars on eigenfunctions of quantum systems with classical analog has also gained a lot of attention.

In the present contribution we begin by generalizing the semiclassical expansion [1] for $\mathrm{n}$-dimensional system. The semiclassical expansion is built in a way that the first order wave function contains the classical dynamics for the system in question as completely as possible, in the sense that the dominant term is given only in terms of classical trajectories. Higher order contributions contain essentially quantum effects and make possible a precise identification of a classical behavior in the quantum dynamics for short times. We use this expansion to obtain the semiclassical Husimi distributions of simple systems.

This paper is organized as follows. In Sections 2 and 3 we present the method, we closely follow ref. [1]. In
Section 4 we present a method of determining a semiclassical Husimi function. Section 7 contains conclusions.

\section{The Semiclassical Expansion}

Let us consider a classical one degree of freedom Hamiltonian of the form

$$
H_{\mathrm{cls}}=\frac{p^{2}}{2 m}+V(q),
$$

where $p$ stands for the particle momentum and $q$ for its position. We make a change of variables

$$
p=\frac{\alpha-\alpha^{*}}{i \sqrt{\frac{2}{m \omega \hbar}}} ; \quad q=\frac{\alpha+\alpha^{*}}{\sqrt{\frac{2 m \omega}{\hbar}}},
$$

where

$$
\begin{aligned}
& \omega=\sqrt{k / m}, \\
& k=\left.\frac{\partial^{2} V(q)}{\partial q^{2}}\right|_{q=0} .
\end{aligned}
$$

The Hamiltonian can then be rewritten as

$$
H_{\mathrm{cls}}=\hbar \omega \alpha^{*} \alpha+U\left(\alpha^{*} \alpha\right)
$$

with $U\left(\alpha^{*} \alpha\right)=V(q)-\left[k\left(\alpha+\alpha^{*}\right) / \sqrt{2 m \omega / \hbar}\right]^{2}$.

We can write $H_{\mathrm{cls}}$ as a Taylor expansion,

$$
H_{\mathrm{cls}}=\hbar \omega \alpha^{*} \alpha+\sum_{m, n} A_{m, n}\left(\alpha^{*}\right)^{m} \alpha^{n},
$$

where $A_{1,1}=0$.

The classical equations of motions are 


$$
\begin{aligned}
& \frac{\mathrm{d}}{\mathrm{d} t} \alpha=\frac{1}{i \hbar} \frac{\partial H_{\mathrm{cls}}}{\partial \alpha^{*}}=-i \omega \alpha-\frac{i}{\hbar} \sum_{m, n} m A_{m, n}\left(\alpha^{*}\right)^{m-1} \alpha^{n} \\
& \frac{\mathrm{d}}{\mathrm{d} t} \alpha^{*}=-\frac{1}{i \hbar} \frac{\partial H_{\mathrm{cls}}}{\partial \alpha}=i \omega \alpha^{*}+\frac{i}{\hbar} \sum_{m, n} n A_{m, n}\left(\alpha^{*}\right)^{m} \alpha^{n-1} .
\end{aligned}
$$

We choose the quantum Hamiltonian $\hat{H}_{q}$ in order to have $\left\langle\alpha\left|\hat{H}_{q}\right| \alpha\right\rangle=H_{\text {cls }}$, if $|\alpha\rangle$ is a coherent field state.

We make our semiclassical expansion around a quantum operator $\hat{H}_{s c}(\alpha(t))$. The difference

$$
\hat{H}_{q}-\hat{H}_{s c}(\alpha(t))=\hat{\delta}(\alpha, \beta, t)
$$

will be considered as a perturbation. We choose the semiclassical Hamiltonian, $\hat{H}_{s c}(\alpha(t))$, in a way that for a coherent initial state, all expectation values of point classical observables will be precisely reproduced.

The semiclassical Hamiltonian which satisfies this condition is [1]:

$$
\begin{aligned}
& \hat{H}_{\mathrm{sc}}(\alpha(t)) \\
= & \hbar \omega \hat{a}^{\dagger} \hat{a}+\sum_{m \neq 0} m A_{m, m}\left(\alpha^{*}\right)^{m-1} \alpha^{m-1}\left(\hat{a}^{?} \hat{a}-\alpha^{*} \alpha\right) \\
& +\sum_{m, n} A_{m, n}\left(\alpha^{*}\right)^{m} \alpha^{n}+\sum_{m \neq n} m A_{m, n}\left(\alpha^{*}\right)^{m-1} \alpha^{n}\left(\hat{a}^{\dagger}-\alpha^{*}\right) \\
& +\sum_{m, n} n A_{m, n}\left(\alpha^{*}\right)^{m} \alpha^{n-1}(\hat{a}-\alpha) .
\end{aligned}
$$

We can write the semiclassical evolution operator for an one degree of freedom, observing that

$$
\begin{aligned}
& \hat{U}_{s c}(\alpha(t))\left|\alpha_{0}\right\rangle=e^{i \phi(t)}|\alpha(t)\rangle \\
& \Rightarrow \hat{U}_{s c}(\alpha(t))=e^{i \phi(t)} e^{i \Omega(t) \hat{a}^{\dagger} \hat{a}} \widehat{D}(\alpha(t)) \widehat{D}\left(\alpha_{0}\right)^{-1}
\end{aligned}
$$

where $\widehat{D}$ is the well known displacement operator

$$
\widehat{D}(\beta)=e^{\beta \hat{a}^{\dagger}-\beta^{*} \hat{a}},
$$

and $\alpha(t)$ is given by 5,6 and $\alpha_{0}=\alpha(0)$.

Thus, for the $\mathrm{N}$ dimensional case we have

$$
\begin{aligned}
\hat{U}_{s c}(\overline{\alpha(t)}) & =\prod_{j}^{N} e^{i \phi(t)} e^{\alpha_{j}(t) \hat{a}_{j}^{\dagger}-\alpha_{j}^{*}(t) \hat{a}_{j}} e^{-\alpha_{j} \hat{a}_{j}^{\dagger}+\alpha_{j}^{*} \hat{a}_{j}} . \\
& =\prod_{j}^{N} \hat{U}_{s c}^{j}\left(\alpha_{j}(t)\right) .
\end{aligned}
$$

where, $\hat{U}_{s c}(\overline{\alpha(t)})=\hat{U}_{s c}\left(\alpha_{1}(t), \alpha_{2}(t) \cdots \alpha_{N}(t)\right)$, and $\hat{U}_{s c}^{j}$ is the semiclassical evolution operator related to the $\mathrm{k}$-th degree of freedom, note that it depends solely on $\alpha_{j}(t)$ but in general we have

$$
\alpha_{j}(t)=f\left(\alpha_{1}(t), \alpha_{2}(t) \cdots \alpha_{N}(t)\right) .
$$

The phase $\phi(\alpha(t))_{j}$ is given by

$$
\phi(\alpha(t))_{j}=-\frac{i}{\hbar} \int_{0}^{t} \mathcal{L}\left[\alpha, \alpha^{*}\right] \mathrm{d} t^{\prime}
$$

where $\mathcal{L}\left[\alpha, \alpha^{*}\right]$ is the classical Lagrangian of the (in- dependent) systems. In equation (9) we chose $\omega_{j}(t)=$ $0^{1}$, what can be done choosing a specific form of the semiclassical Hamiltonian, see [1]. A generalization for SU(2) algebra or for any subspace where coherent states can be included, is immediate. The action of the semiclassical evolution operator over a coherent state can always be written as [17]

$$
\hat{U}_{s c}^{j}(\alpha(t))\left|\beta_{j}\right\rangle=e^{i \rho_{j}(t)}\left|\alpha_{j}(t)+\beta_{j}-\alpha_{j}(0)\right\rangle
$$

where

$$
\begin{aligned}
\rho_{j}\left(\alpha_{j}(t), \beta_{j}\right)= & \phi_{j}(\alpha(t))-\operatorname{Im}\left(\alpha_{j}(t) \beta_{j}^{*}\right) \\
& +\operatorname{Im}\left[\left(\alpha_{j}(0)-\beta_{j}\right)^{*} \alpha(t)\right] .
\end{aligned}
$$

In general $\alpha_{k}(t)$ is a function of all $\alpha_{j}(t)$. In the next sections we use the fact that the labels of coherent states follow the classical trajectories.

\section{Time Evolution}

We consider a two degrees of freedom system, which the complete Hamiltonian is given by

$$
\widehat{H}_{q}=\widehat{H}_{1}+\widehat{H}_{2}+\widehat{H}_{12}
$$

where $\widehat{H}_{1(2)}$ represent the autonomous dynamics of the degree of freedom 1 (2) and $\widehat{H}_{12}$ is their interaction. The semiclassical Hamiltonian has the following form ${ }^{2}$

$$
\begin{aligned}
& \widehat{H}_{s c}(\alpha(t), \beta(t), t) \\
= & \widehat{H}_{s c}^{1}(\alpha(t), \beta(t), t)+\widehat{H}_{s c}^{2}(\alpha(t), \beta(t), t)+H_{c l},
\end{aligned}
$$

and by definition we have

$$
\left[\widehat{H}_{s c}^{1}(\alpha(t), \beta(t), t), \widehat{H}_{s c}^{2}(\alpha(t), \beta(t), t)\right]=0 .
$$

As discussed in section II we rewrite the Hamiltonian (12) in the following form $\widehat{H}=\widehat{H}_{s c}(\alpha, \beta, t)+\hat{\delta}(\alpha, \beta, t)$, where $\hat{\delta}(\alpha, \beta, t)=\widehat{H}-\widehat{H}_{s c}(\alpha, \beta, t)$. We make a perturbation expansion about $\hat{\delta}(\alpha, \beta, t)$. Using Schrödinger's equation, where we will always use as initial state $|\Psi(0)\rangle=|\alpha(0)\rangle|\beta(0)\rangle, \quad|\alpha(0)\rangle$ and $|\beta(0)\rangle$ are coherent states. Thus, after some straightforward algebraic manipulations [1] we get

$$
\begin{aligned}
|\Psi\rangle(t) & =\hat{U}_{s c}(\alpha, \beta, t)\left\{1+\frac{1}{i \hbar} \int_{0}^{t} \hat{\Delta}_{s}(t) \mathrm{d} t_{1}\right. \\
& \left.+\left(\frac{1}{i \hbar}\right)^{2} \int_{0}^{t} \int_{0}^{t_{1}} \hat{\Delta}_{s}\left(t_{1}\right) \hat{\Delta}_{s}\left(t_{2}\right) \mathrm{d} t_{1} \mathrm{~d} t_{2}+\cdots\right\}|\Psi\rangle(0)
\end{aligned}
$$

\footnotetext{
${ }^{1} \omega_{j}$ refers to j-th system.

${ }^{2} \widehat{H}_{s c}^{1}(\alpha, \beta, t)$ represents the semiclassical Hamiltonian in the subspace 1, and $\widehat{H}_{s c}(\alpha, \beta, t)$ represents the semiclassical Hamiltonian in the subspace 2 .
} 
where $\hat{\Delta}_{s}(t, \alpha, \beta)=\hat{U}_{s c}^{\dagger}(\alpha, \beta, t) \hat{\delta}(\alpha, \beta, t) \hat{U}_{s c}(\alpha, \beta, t)$. The general problem of convergence of the serie (14) is an open problem. The convergence of the method was demonstrated for the quartic oscilator [1] and there is strong evidence of it for chaotic Dikie model [18], then it is reasonable to assume that the method is convergent at least to a group of non-integrable systems.

\section{Husimi’s Quantum Phase Space Distribution}

The Q-function or Husimi's function is, see refs. [4,19], defined by:

$$
\mathbf{H}_{\Psi}(Q, P)=\left\langle\alpha\left|\rho_{\Psi}\right| \alpha\right\rangle,
$$

$\rho_{\Psi}$ is a density operator, and $|\alpha\rangle$ is the harmonic coherent state according to the definitions:

$$
\begin{aligned}
& Q=\frac{\langle\hat{q}\rangle}{\beta}, P=\frac{\langle\hat{p}\rangle}{\hbar} \beta \\
& \beta=\sqrt{\frac{\hbar}{m \omega}}, \alpha=\frac{Q+i P}{\sqrt{2}} .
\end{aligned}
$$

$q$ and $p$ are position and momentum operator respectively, and the mean is calculated in the coherent state $|\alpha\rangle$. From this definition, we are able to write the Husimi function as

$$
\mathbf{H}_{\Phi}(Q, P)=\left|\iiint \Phi(x, y, z) \chi(x, y, z) \mathrm{d} x \mathrm{~d} y \mathrm{~d} z\right|^{2} .
$$

$\Phi(x, y, z)$ is the system Hamiltonian eigenfunction, and $\chi(x, y, z)$ is the harmonic coherent state in three dimensions, it is given by

$$
\chi_{\alpha}(x, y, z)=\prod_{i=1}^{3} \mu_{\theta_{i}} \exp \left[-\left(\frac{x_{i}-\left\langle x_{i}\right\rangle_{\alpha_{i}}}{2 \Delta x}\right)^{2}+i\left\langle p_{x_{i}}\right\rangle_{\alpha_{i}} \frac{x_{i}}{\hbar}\right],
$$

where $x_{1}=x, x_{2}=y, x_{3}=z, \quad p_{x_{i}}$ is the momentum related with $x_{i}$, and

$$
\begin{aligned}
& \mu_{\theta_{i}}=e^{\frac{\alpha_{i}^{* 2}-\alpha_{i}^{2}}{4}}\left(\frac{\mu \omega}{\pi \hbar}\right)^{\frac{1}{4}}, \\
& \Delta x=\Delta y=\Delta z=\sqrt{\frac{\hbar}{2 \mu \omega}} .
\end{aligned}
$$

For the simplest case of the Harmonic Oscillator, using equation [15], the Husimi Function for an eigenstate, $n$, can be written as:

$$
\mathbf{H}_{n}(\alpha)=\frac{e^{-|\alpha|^{2}}|\alpha|^{2 n}}{(2 \pi \hbar) n !}
$$

In terms of $Q$ and $P$, we have

$$
\mathbf{H}_{n}(Q, P)=\frac{e^{-\left(Q^{2}+P^{2}\right) / 2}\left(Q^{2}+P^{2}\right)^{n}}{(4 \pi \hbar) n !}
$$

\subsection{Husimi Function for the Morse Potential}

The Morse potential is used to model diatomic molecules, it is defined as :

where

$$
\begin{gathered}
U(x)=D\left(e^{-2 \eta x}-2 e^{-\eta x}\right), \\
x \equiv \frac{r-r_{0}}{r_{0}} .
\end{gathered}
$$

The $r_{0}$ values are the equilibrium position of the center of mass, $\mu$ is the reduced mass of the two atoms and $\mathrm{r}$ is the distance between the atoms. The constant $D$ defines the minimum value o the potential wich is $-D$. The constant $\eta$ determines the potential range. The Hamiltonian that describes the center of mass can be written as:

$$
H=\frac{1}{2} \mu\left(\frac{\mathrm{d} r}{\mathrm{~d} t}\right)^{2}+\frac{1}{2} \frac{L^{2}}{\mu r^{2}}+U(r)
$$

where $L$ is the angular momentum. The time independent Schrödinger equation is:

$$
\left[-\frac{\hbar^{2}}{2 \mu} \frac{1}{r} \frac{\partial^{2}}{\partial r^{2}} r+\frac{L^{2}}{2 \mu r^{2}}+V(r)\right] \Phi(r, \theta, \varphi)=E \Phi(r, \theta, \varphi) \text {. }
$$

We can write the wavefunction as

$$
\Phi(r, \theta, \varphi)=\frac{\Psi(r)}{r} Y_{l m}(\theta, \varphi),
$$

where $Y_{I m}$ is the spherical harmonics:

$$
Y_{l}^{m}(\theta, \varphi)=A_{l m} P_{l}^{m}[\cos (\theta)] e^{i m \varphi} .
$$

For $L=0$ case we find the eigenvalues:

$$
E_{v}=-D+\hbar \omega\left(v+\frac{1}{2}\right)-\frac{1}{\zeta}\left(v+\frac{1}{2}\right)^{2}
$$

where $v=0,1,2,3, \cdots \quad v<\frac{1}{2}(\zeta-1)$, and

$$
\begin{aligned}
& \beta^{2}=-\frac{2 \mu E_{v} r_{0}^{2}}{\hbar^{2}}, \quad \gamma^{2}=\frac{2 \mu D r_{0}^{2}}{\hbar^{2}}, \\
& \varsigma=\frac{2 \beta}{\eta}+1, \quad \tilde{a}=\frac{1}{2} \varsigma-\frac{\gamma}{\eta}, \zeta=\frac{2 \gamma}{\eta} .
\end{aligned}
$$

and for the eigenfunctions:

$$
\begin{aligned}
\frac{\Psi(x)}{r}= & \frac{A_{1}}{r_{0}(x+1)} \frac{\Gamma(\varsigma)}{\Gamma(\tilde{a})} \exp \left(-\beta x-\frac{1}{2} \zeta e^{-\eta x}\right) \\
& \times \sum_{n=0}^{\infty}\left[\frac{\Gamma(\tilde{a}+n)}{\Gamma(\varsigma+n)} \frac{\left(\zeta e^{-\eta x}\right)^{n}}{n !}\right] .
\end{aligned}
$$


$\mathrm{A}_{1}$ is fixed by normalization, $\Gamma(x)$ is the gamma function.

Following the definition (20), we obtain the Husimi the Function [20] as

$$
\mathbf{H}_{\Phi}(Q, P)=\left|2 \pi \int_{-1}^{\infty} I_{\theta 0}(x) \chi(x) \Psi(x) r_{0}^{2}(x+1) \mathrm{d} x\right|^{2},
$$

where $I_{\theta 0}(r)=\frac{1}{\sqrt{\pi}} \frac{\sinh \left(\alpha_{0} r\right)}{\alpha_{0} r}, l=0$. The exact Husimi function is obtained by numerically integration of (33).

\subsection{Semiclassical Husimi’s Function}

The semiclassical expansion, as defined above, gives us the time evolution of a quantum state as a perturbative expansion. An eigenstate has only a time dependent phase as its dynamics. The nearest semiclassical scenario we can build is to choose a coherent state with the same energy. The time dependence can be eliminated by a time integration, i.e., a mean in time. This integration can be justified noting that as we are dealing with eigenstates we have not time precision. Under this considerations we may write the semiclassical Husimi function as

$$
\mathbf{H}_{s c}(\beta)=\overline{\langle\beta \mid \alpha(t)\rangle}^{2} .
$$

The states $|\alpha(t)\rangle$ and $|\beta\rangle$ are coherent states of the harmonic oscillator. $\alpha(t)$ is defined as

$$
\alpha(t)=\frac{Q(t)+i P(t)}{2} \text { and } \beta=x+i p_{x},
$$

where $x$ and $p_{x}$ are parameters of the Husimi Function, $Q(t)$ and $P(t)$ correspond to the classical canonical conjugate pairs redefined as in Equations (16) and (17). In case of classical mixed dynamics we must perform a mean considering all possible initial condition with the same specific energy. We should also use adequate coherent state base for each algebra. This semiclassical Husimi function is calculated by taking a long time mean, formally we write it as

$$
\mathbf{H}_{s c}(\beta)=\lim _{\tau \rightarrow \infty} \frac{\int_{0}^{\tau}|\langle\beta \mid \alpha(t)\rangle|^{2} \mathrm{~d} t}{\tau} .
$$

As we known, classically chaotic systems stay longer times [4] near a stable or unstable periodic orbit. In our numerical calculation it means that this region has a huge contribution in the mean (35) or (34). Easily we can show that

$$
\mathbf{H}_{s c}(\beta)=\overline{e^{|Q(t)+i P(t)-\beta|^{2}}} .
$$

The semiclassical Husimi distributions is determined by the classical trajectory solely. In general the semiclassical Husimi function is obtained by numerical methods, it is the case of all chaotic model.

\subsubsection{Morse Potential}

For the Morse potential, with $L=0$, we obtain the classical trajectory [20] as:

$$
Q(t)=\frac{1}{\alpha} \ln \left\{\frac{D}{|E|}+\left[\sin \left(t \sqrt{\frac{2|E| \alpha^{2}}{\mu r_{0}^{2}}}\right) \sqrt{\frac{D^{2}+E D}{|E|^{2}}}\right]\right\} E<0
$$

We also have $P(t)=\mu \frac{\mathrm{d} Q(t)}{\mathrm{d} t}$, and we can choose $p(0)=0$ and choosing the energy as $E=E_{v}$ into (36) to obtain the semiclassical Husimi function. The mean (35) is obtained by a numerical integration. In Figure 1 we show the approximated semiclassical Husimi for the Morse potential with the parameters of the $\mathrm{H}_{2}$ molecule, for $v=0$. In Figure 2 we have the exact result, Figure 3 shows the semiclassical Husimi function for $n=$ 1 and Figure 4 the exact result, details about exact calculation can be found in ref. [20]. As we can observe in this Figures 1-4, the semiclassical Husimi function does not reproduce exactly the Husimi function, but it regards some similarities. Notice that the main region is located in the same phase space area for the exact and semiclassical Husimi function.

\subsubsection{Harmonic Oscillator}

Now consider the Harmonic potential with a natural frequency $\omega$, its classical dynamics is given by

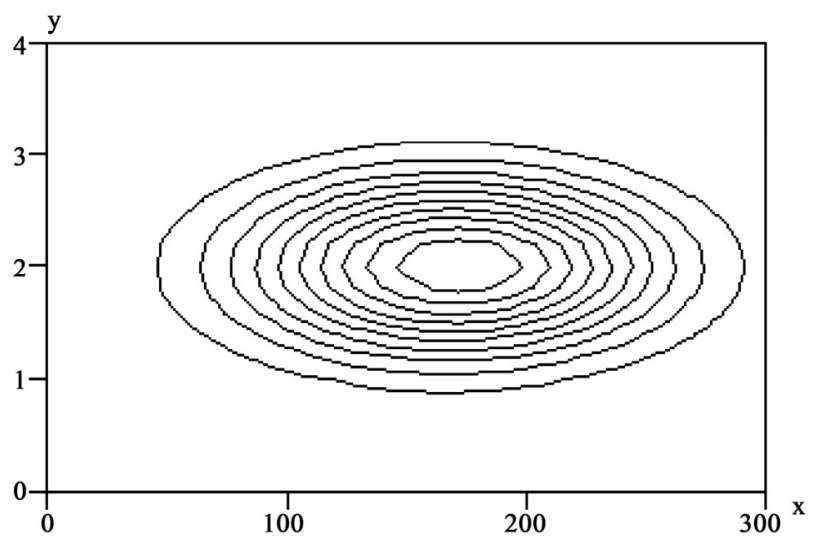

Figure 1. Semiclassical Husimi function for the Morse Potential, $U(x)=D\left(e^{-2 \alpha x}-2 e^{-\alpha x}\right)$, where $x=\frac{r-r_{0}}{r}$. We have used the experimental hydrogen molecule values, $\alpha=1.440$ and $D=4.75(\mathrm{eV})$. The principal quantum number $\mathrm{n}=0$ and the angular momentum $L=0$. The $\mathrm{x}$ axis is related to the position, $r$, as $r=r_{0} \frac{(x+10)}{250}$, the momentum $p$ is related to the $y$ axis variable as $p=m \omega r_{0} \frac{(y-2)}{1.2}$. We have used $r_{0}=7.42 \times 10^{-11} \mathrm{~m}$ and $\omega=8.3 \times 10^{14} \mathrm{rad} / \mathrm{s}$. 


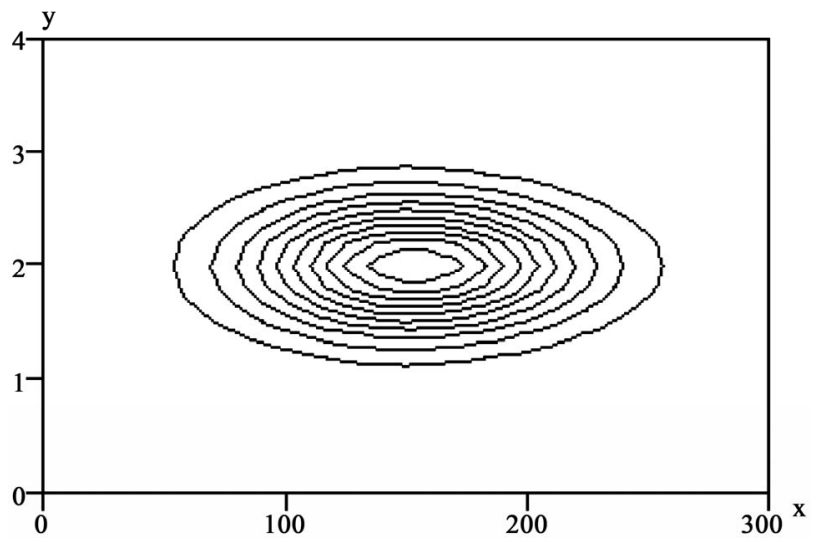

Figure 2. Husimi function for the Morse potential with the same parameters of Figure 1.

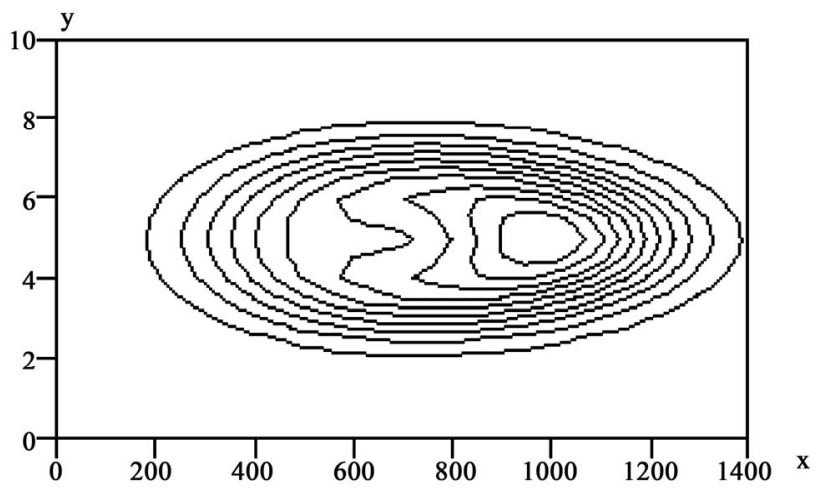

Figure 3. Semiclassical Husimi function for the Morse

Potential, $U(x)=D\left(e^{-2 \alpha x}-2 e^{-\alpha x}\right)$, where $x=\frac{r-r_{0}}{r_{o}}$. We have used the experimental hydrogen molecule values, $\alpha=1.440$ and $D=4.75(\mathrm{eV})$. The principal quantum number $n=1$ and the angular momentum $L=0$. The $x$ axis is related to the position, $r$, as $r=r_{0} \frac{(x+300)}{1000}$, the momentum $p$ is related to the $y$ axis variable as $p=m \omega r_{0} \frac{(y-5)}{5}$. We have used $r_{0}=7.42 \times 10^{-11} \mathrm{~m}$ and $\omega=8.3 \times 10^{14} \mathrm{rad} / \mathrm{s}$.

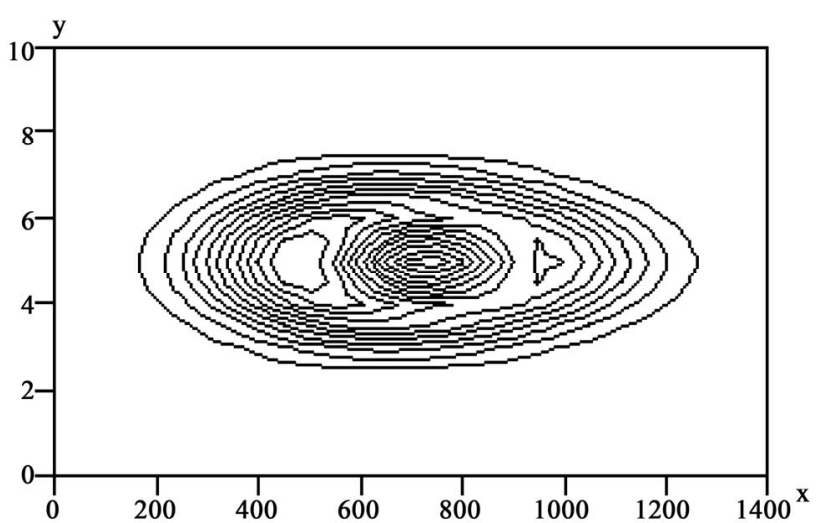

Figure 4. Husimi function for the Morse potential with the same parameters of Figure 3.

$$
Q(t)=Q(0) \cos (\omega t)+\frac{P(0)}{\omega} \sin (\omega t)
$$

and

$$
P(t)=P(0) \cos (\omega t)-Q(0) \omega \sin (\omega t)
$$

We redefined $Q$ and $P$ in a such way that the Hamiltonian can be written as

$$
H=\hbar \omega \frac{\left(Q^{2}+P^{2}\right)}{2} .
$$

Substituting (38) and (39) into (36) we obtain a semiclassical Husimi Function for the Harmonic oscillator with an energy $E_{n}=\hbar \omega\left(n+\frac{1}{2}\right)$. Without any loss of generality we can use $Q(0)=\left(2 E_{n}\right)^{1 / 2}$, and $P(0)=0$. In Figure 5 we show the approximated and exact Husimi function for the Harmonic potential for $n=5$. Figure 6 shows the semiclassical and exact Husimi function for $n=100$. Again the mean (35) is obtained numerically.

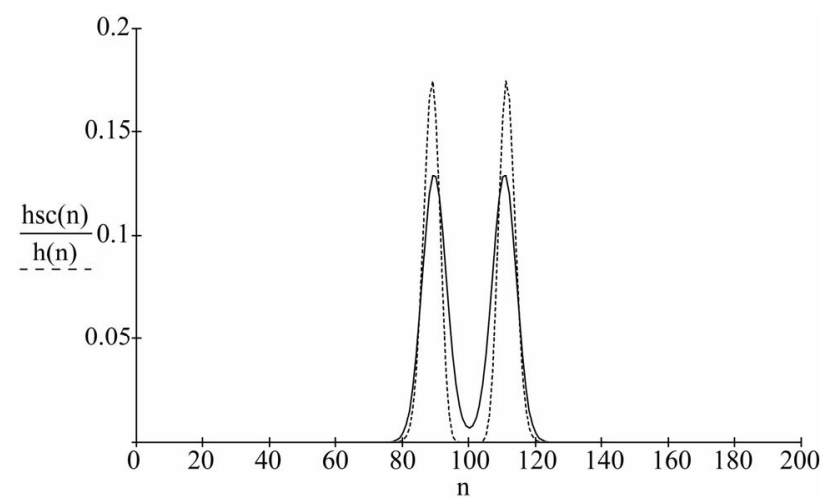

Figure 5. Full line shows a cross section of Husimi function for the harmonic potential for $p=0$. The principal quantum number $n=5$. The $x$ axis is related to the position. Dotted line, correspondent semiclassical Husimi function.

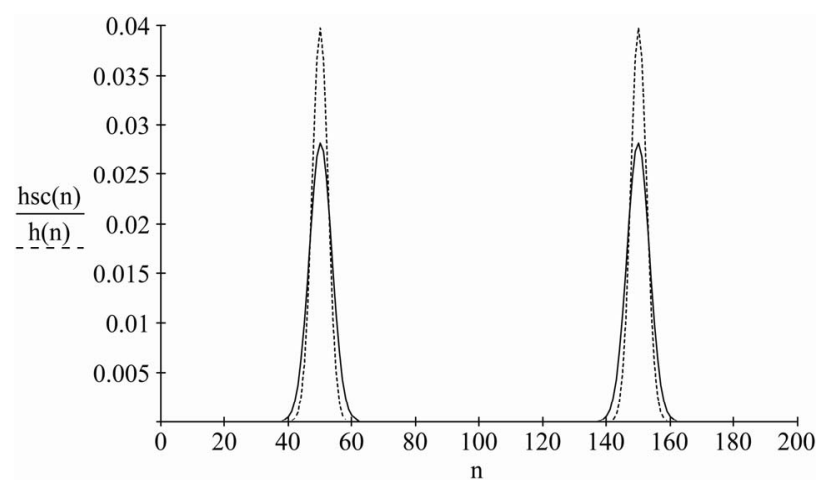

Figure 6. Full line shows a cross section of Husimi function for the harmonic potential for $p=0$. The principal quantum number $n=100$. The $x$ axis is related to the position. Dotted line, correspondent semiclassical Husimi function. 


\subsubsection{Dickie Model}

In the Figures 1 to 7 we have used classical integrable models, although that our approach is also useful for non-integrable thus let us take a look in Dikie model [21], his quantum Hamiltonian is

$$
H=H_{O}+H_{G^{\prime}}+H_{G},
$$

where

$$
\begin{gathered}
H_{O}=\hbar \omega \hat{a}^{\dagger} \hat{a}+\hbar \varepsilon \hat{J}_{z}, \\
H_{G^{\prime}}=\frac{G^{\prime}}{\sqrt{2 J}}\left(\hat{a} \hat{J}_{-}+\hat{a}^{\dagger} \hat{J}_{+}\right), \\
H_{G}=\frac{G}{\sqrt{2 J}}\left(\hat{a} \hat{J}_{+}+\hat{a}^{\dagger} \hat{J}_{-}\right),
\end{gathered}
$$

$\omega$ is the harmonic oscillator natural frequency, $\varepsilon$ is precession frequency, $G$ and $G^{\prime}$ are coupling constants. In the harmonic term of (42) $\hat{a}$ and $\hat{a}^{\dagger}$ are bosonic anihilation and creation operators of harmonic oscillator, $\hat{J}_{k}$ is the angular momentum operator in $k$ direction and $\hat{J}_{ \pm}=\hat{J}_{x} \pm \hat{J}_{y}$. In order to obtain the Semiclassical Husimi function for the Dickie model we have to integrate numerically the corresponding classical equations of motion and calculate the mean (35).

In Figure 7 we show the semiclassical Wigner function of ground state of Dickie model [21] in an integrable regime. In Figure 8 we have the semiclassical Wigner function for Dickie model in a non-integrable regime. Comparing with exact Wigner $^{3}$ function that can be found in ref. [21], we see that semiclassical Husimi function contains the main information about the exact one. A detailed semiclassical analysis of Dickie's model can be found in $[18,22]$. In [18] they show a numerical evidence of the expansion convergence for the Dickie's model.

\subsubsection{The Quality of the Approximation}

Now we quantify the quality of the approximation using the function $\Delta \mathbf{Q}_{\psi_{n}}(q)$, which is defined as

$$
\Delta \mathbf{Q}_{\psi_{n}}(q)=\mathbf{H}(\mathbf{q}, \mathbf{p}=\mathbf{0})-\mathbf{H}(\mathbf{q}, \mathbf{p}=\mathbf{0})_{s c},
$$

where $\mathbf{H}$ is the exact Husimi function and $\mathbf{H}_{s c}$ is the semiclassical Husimi function. Due to the symmetry we have chosen $p=0$. As we increase the principal quantum number (n), in the classical limit, we hope we have $\Delta \mathbf{Q}_{\psi_{n}}(q) \rightarrow 0$. In order to see the classical limit, let us define the function $S Q_{\psi_{n}}$, which is

$$
S Q_{\psi_{n}}=\int_{-\infty}^{\infty}\left|\Delta Q_{\psi_{n}}(q)\right| \mathrm{d} q
$$

\footnotetext{
${ }^{3}$ For stationary states semiclassical Wigner function and semiclassical Hunimi function are identical.

${ }^{4}$ For example, the Wigner function can be negative at some point while the semiclassical Husimi function and the exact one are almost identical.
}

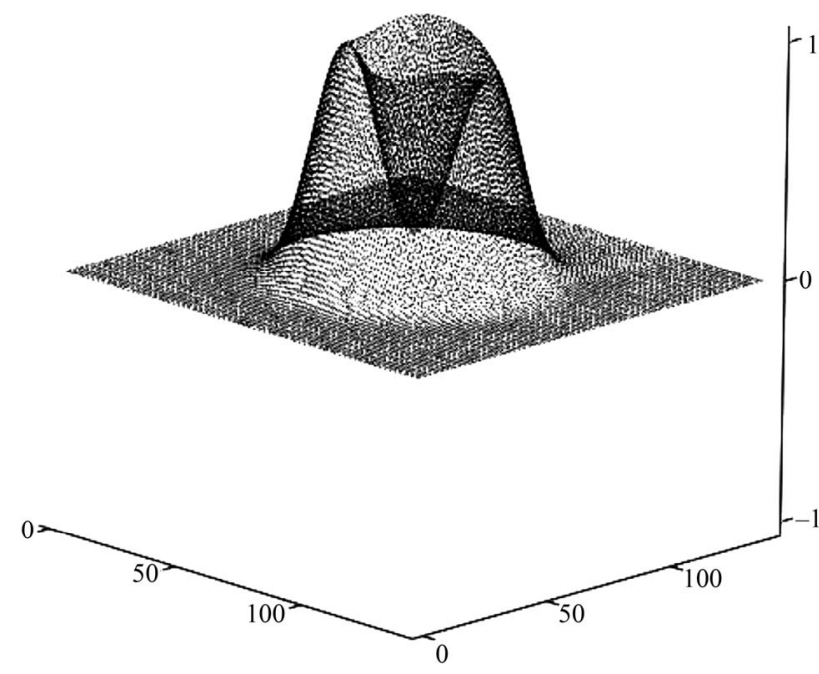

Figure 7. Semiclassical Wigner function for the fundamental state of Dickie model where $G^{\prime}=0, \lambda=1.5, \omega=1$.

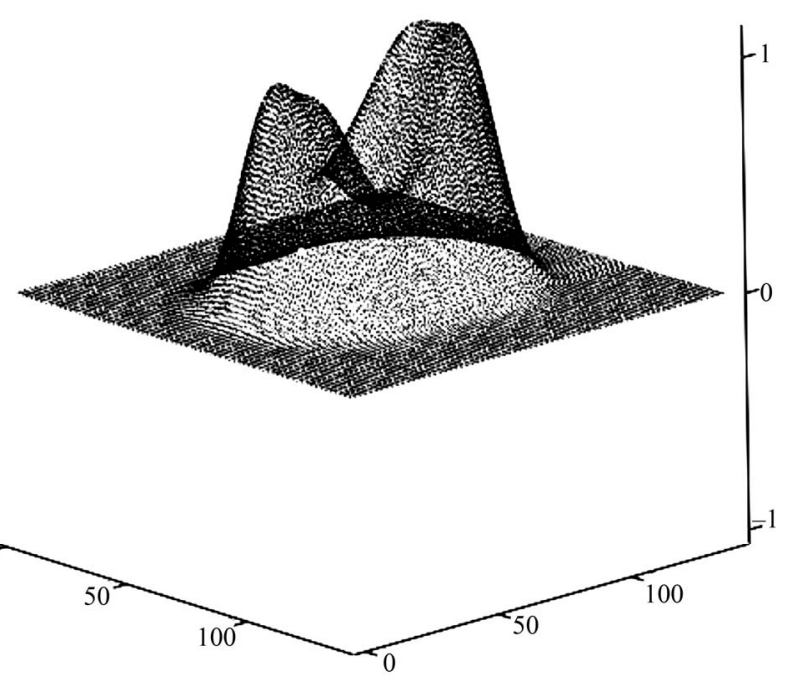

Figure 8. Semiclassical Wigner function for the fundamental state of Dickie model $G=G^{\prime}=0.75$.

Suppose we have $S Q_{\psi_{n}} \approx 0$. It means that quantum description of the state $\psi$, in the Husimi's representation, is almost contained in the semiclassical one. In spite of that we can say that the quantum classical difference becomes smaller, as expected. Of course it does not mean that we have no quantum features ${ }^{4}$, it only means that Husimi is not a good observable for this situation [23]. Figure 9 shows $S Q_{\psi_{n}}$ say that the approximation works better as we increase the principal quantum number, as expected. From these figures we may conclude that the classical ingredient is very strong on the state formation of regular systems. In other words, those figures suggests that scars are essentially a classical manifestation in the quantum system since the building blocks of the semiclassical Husimi are classical trajectories. 


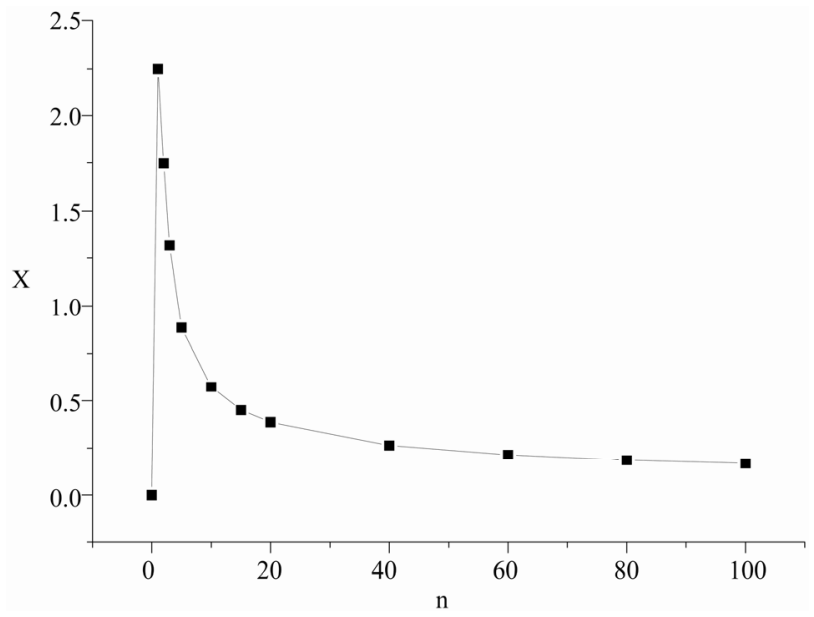

Figure 9. SQ function for the harmonic oscillator. $\mathrm{X}$ axis corresponds to the principal quantum number $n$.

\section{Conclusion}

We show that the semiclassical Husimi function reproduces the major features of the quantum one, in the particular harmonic case, we show that the first semiclassical term is able to reproduce the Husimi function with a increasing accuracy as we increase the principal quantum number $n$. We must remark that there is no demonstration that would suggest an existence of the limit procedure which turns quantum corrections less important in terms of the proposed semiclassical expansion. The building blocks of semiclassical Husimi Function are the classical trajectories, then we can conclude that classical periodic orbits (stable or unstable) contribute with higher weight. The fidelity decay rate has a gaussian regime that is only perturbation potential dependent, although its validity is determined by $t<\tau_{s c}$.

\section{Acknowledgements}

The author is grateful to Fapesb for partial financial support. The author also acknowledge, A. R. Bosco de Magalhaes, M. C. Nemes, Fernanda Alves de Oliveira for helpful comments.

\section{REFERENCES}

[1] A. C. Oliveira and M. C. Nemes and K. M. F. Romero, "Quantum Time Scales and the Classical Limit: Analytic Results for Some Simple Systems," Physical Review E, Vol. 68, No. 3, 2003, Article ID: 036214. doi:10.1103/PhysRevE.68.036214

[2] A. Einstein, "Zum Quantensatz von Sommerfeld und Epstein on the Quantum Theory of Sommerfeld and Epstein," Deutsche Physikalische Gesellschaft Verhandlungen, Vol. 19, 1917, p. 82.

[3] M. A. M. de Aguiar, "Einstein and the Quantum Chaos Theory," Revista de Ensino de Física, Vol. 27, 2005, p.
101.

[4] M. C. Gutzwiller, "Chaos in Classical and Quantum Mechanics," Spring-Verlg, New York, 1990.

[5] H. J. StockmannHaake, "Quantum Chaos an Introduction," Cambridge University Press, New York, 1999.

[6] F. Haake, "Quantum Signatures of Chaos," SpringerVerlag, Berlin, 2004.

[7] S. W. McDonald and A. N. Kaufmann, "Spectrum and Eigenfunctions for a Hamiltonian with Stochastic Trajectories," Physical Review Letters, Vol. 42, No. 18, 1979 pp. 1189-1191. doi:10.1103/PhysRevLett.42.1189

[8] E. B. Bogomolny, "Fine Structure of the Wave Functions of Quantum Systems," JETP Letters, Vol. 44, No. 9, 1986, pp. 561-565.

[9] E. B. Bogomolny, "Smoothed Wave Functions of Chaotic Quantum Systems," Physica D, Vol. 31, No. 2, 1988, pp. 169-189. doi:10.1016/0167-2789(88)90075-9

[10] E. J. Heller, "Bound-State Eigenfunctions of Classically Chaotic Hamiltonian Systems: Scars of Periodic Orbits," Physical Review Letters, Vol. 53, No. 16, 1984, pp. 15151518. doi:10.1103/PhysRevLett.53.1515

[11] E. J. Heller, "Quantum Chaos and Statistical Nuclear Physics,” Springer, Berlin, 1983.

[12] L. Benet and T. H. Seligman and H. A. Weidenmuller, "Quantum Signatures of Classical Chaos: Sensitivity of Wave Functions to Perturbations," Physical Review Letters, Vol. 71, No. 4, 1993, pp. 529-532. doi:10.1103/PhysRevLett.71.529

[13] M. Srednicki and F. Stiernelof, "Gaussian Fluctuations in Chaotic Eigenstates," Journal of Physics A, Vol. 29, No. 18, 1996, p. 5817. doi:10.1088/0305-4470/29/18/013

[14] L. Benet and F. M. Izrailev and T. H. Seligman and A. Suarez-Moreno, "Semiclassical Properties of Eigenfunctions and Occupation Number Distribution for a Model of Two Interacting Particles," Physics Letters A, Vol. 277, No. 2, 2000, pp. 87-93. doi:10.1016/S0375-9601(00)00692-7

[15] L. Benet, et al., "Fluctuations of Wavefunctions about Their Classical Average," Journal of Physics A, Vol. 36, No. 5, 2003, p. 1289. doi:10.1088/0305-4470/36/5/307

[16] P. Bellomo and T. Uzer, "Quantum Scars and Classical Ghosts," Physical Review A, Vol. 51, No. 2, 1995, pp. 1669-1672. doi:10.1103/PhysRevA.51.1669

[17] W. Zhang, H. Feng and R. Gilmore, "Coherent States: Theory and Some Applications," Reviews of Modern Physics, Vol. 62, No. 4, 1990, pp. 867-927. doi:10.1103/RevModPhys.62.867

[18] M. Reis and M. C. Nemes and J. G. P. de Faria, "Semiclassical Corrections to the Large- $N$ Limit of Dicke's Model," Physical Review E, Vol. 78, No. 3, 2008, Article ID: 036220. doi:10.1103/PhysRevE.78.036220

[19] W. P Scleichr, "Quantum Optics in Pahse Space," WileyVCC, Berlin, 2001.

[20] A. C. Oliveira and M. C. Nemes, "Classical Structures in the Husimi Distributions of Stationary States for $\mathrm{H}_{2}$ and $\mathrm{HCl}$ Molecules in the Morse Potential," Physica Scripta, Vol. 64, No. 4, 2001, p. 279. 
doi:10.1238/Physica.Regular.064a00279

[21] M. C. Nemes, K. Furuya, G. Q. Pelegrino, A. C. Oliveira, M. Reis and L. SanzM, "Quantum Entanglement and Fixed Point Hopf Bifurcation," Physics Letters A, Vol. 354, No. 1-2, 2006, pp. 60-66. doi:10.1016/i.physleta.2006.01.028

[22] K. M. F. Romero, M. C. Nemes, J. G. P. de Faria and A. F. R. de T. Piza, "Sensitivity to Initial Conditions in Quantum Dynamics: An Analytical Semiclassical Expansion,"
Physics Letters A, Vol. 327, No. 2-3, 2004, pp. 129-137. doi:10.1016/i.physleta.2004.04.070

[23] A. C. Oliveira and J. G. P. de Faria and M. C. Nemes, "Quantum-Classical Transition of the Open Quartic Oscillator: The Role of the Environment," Physical Review E, Vol. 73, No. 4, 2006, Article ID: 046207. doi:10.1103/PhysRevE.73.046207 